\title{
Erratum to: Abstracts of the 2011 South East Asian Congress of Medical Physics (SEACOMP 2011)
}

\author{
John R. Cameron Memorial Lecture
}

\author{
Augusto A. Morales Jr.
}

Published online: 7 May 2013

(C) Australasian College of Physical Scientists and Engineers in Medicine 2013

\section{Erratum to: Australas Phys Eng Sci Med (2012) 35:365-387 \\ DOI 10.1007/s13246-012-0154-0}

In the Conference Abstracts mentioned above, published in Australasian Physical \& Engineering Sciences in Medicine, volume 35, issue 3, September 2012, pp. 365-387, several authors were not mentioned in the author group of abstracts to which they have contributed. Please see the changes below:

\section{Change 1}

From:

Extremely low frequency magnetic field measurements in a residence adjacent to 86 kVA transformers in Brgy, Siena, Quezon City

Joyce Ann T. de Guzman

The Graduate School, University of Santo Tomas, España, Manila, Philippines

To:

Extremely low frequency magnetic field measurements in a residence adjacent to 86 kVA transformers in Brgy, Siena, Quezon City

The online version of the original article can be found under doi: 10.1007/s13246-012-0154-0

The online version of the original article can be found under doi: $10.1007 / \mathrm{s} 13246-012-0154-0$.

A. A. Morales Jr. ( $\square)$

Center for Device Regulation, Radiation Health and Research, FDA Philippines, Department of Health, Rizal Avenue, Manila 1003, Philippines

e-mail: seacomp2011@gmail.com
Joyce Ann T. de Guzman ${ }^{1}$ and Arnold I. Eleazar ${ }^{2}$

${ }^{1}$ The Graduate School, University of Santo Tomas, España, Manila, Philippines

${ }^{2}$ Center for Device Regulation, Radiation Health and Research, Food and Drug Administration, Department of Health, Manila, Philippines

Change 2

From:

Occupational microwave radiation power density levels from the radar facility in Mactan Cebu International Airport (MCIA)

Realyn Joy L. Uy

Center for Device Regulation, Radiation Health and Research, Food and Drug Administration, Manila, Philippines

To:

Occupational microwave radiation power density levels from the radar facility in Mactan Cebu International Airport (MCIA)

Realyn Joy L. Uy ${ }^{1}$ and Agnette P. Peralta ${ }^{2}$

${ }^{1}$ University of Santo Tomas Graduate School, España Boulevard, Manila, Philippines

${ }^{2}$ Center for Device Regulation, Radiation Health and Research, Food and Drug Administration, Department of Health, Manila, Philippines

Change 3

From:

Radio frequency radiation power density levels from selected ultra high frequency (UHF) and very high frequency (VHF) television broadcast transmitters in Metro Manila, Philippines

Annie Lorraine B. Joyas

Center for Device Regulation, Radiation Health and Research, Food and Drug Administration, Manila, Philippines 
To:

Radio frequency radiation power density levels from selected ultra high frequency $(U H F)$ and very high frequency (VHF) television broadcast transmitters in Metro Manila, Philippines

Annie Lorraine B. Joyas ${ }^{1}$ and Arnold I. Eleazar ${ }^{2}$

${ }^{1}$ University of Santo Tomas Graduate School, España Boulevard, Manila, Philippines

${ }^{2}$ Center for Device Regulation, Radiation Health and Research, Food and Drug Administration, Department of Health, Manila, Philippines

\section{Change 4}

From:

Computed tomography tests on some components of image quality for treatment planning system

Daniel D. Vicario ${ }^{1,2}$

${ }^{1}$ Benavides Cancer Institute, University of Santo Tomas Hospital, Manila, Philippines

${ }^{2}$ College of Science, University of Santo Tomas, Manila, Philippines

To:

Computed tomography tests on some components of image quality for treatment planning system

\section{Daniel D. Vicario ${ }^{1,2}$ and Maureen R. Bojador ${ }^{1}$}

${ }^{1}$ Benavides Cancer Institute, University of Santo Tomas Hospital, Manila, Philippines

${ }^{2}$ College of Science, University of Santo Tomas, Manila, Philippines

The authors would also like to add the following abstract:

Evaluation of a Deformable Registration Method for Critical Organs and Target in head and neck patients Jerome Haw Hwong Yap ${ }^{1}$, James Chow Lei Lee ${ }^{1}$, Francis Chin ${ }^{1}$

${ }^{1}$ National Cancer Centre Singapore, Department of Oncology

Introduction Head and neck patients who have dramatic shrinkage of neck nodes during IMRT treatment is not an uncommon clinical scenario and often require re-planning and recontouring mid treatment. Manual contouring of normal organs for retreatment plans for patients is a time consuming process. If this process can be automated, it will result in dramatically shortened re-planning times. We aim to find the most feasible method with this study with the manual contouring of experts as clinical gold standard.

Methods Two CTs were acquired for each of 7 head and neck cancer patients. All contours were generated on Eclipse 8.6. Contours were manually drawn on the initial planning CT ( set A) and re-planning CT (B). The deformable adaptive re-contouring method (MIMvista) was used to generate the automatic contours $(\mathrm{C})$ from set A. Consistency between sets was compared using the contour overlap metric, defined as the proportion of the set intersection to the set union of a contour pair.

Results The overlap index of brainstem, spinal cord, eye, gross tumor volume (GTV), parotid, lens, optic nerve and chiasm are $0.78 \pm 0.04,0.76 \pm 0.06,0.85 \pm 0.03,0.82 \pm$ $0.06,0.75 \pm 0.05,0.43 \pm 0.09,0.52 \pm 0.20$ and $0.32 \pm$ 0.19 respectively. Most of the organs the average overlap index for 7 patients are either 0.75 or more.

Relatively small and irregular shape organ as like chiasm has the lowest overlap index of $0.32 \pm 0.19$.

Discussion Brainstem, spinal cord, eye, GTV and parotid the volume range from $20 \mathrm{cc}$ to $110 \mathrm{cc}$ in this study. While lens, optic nerve and chiasm the volume range from $0.3 \mathrm{cc}$ to $3 \mathrm{cc}$. Organs with small volume all have relatively low overlap index to the rest. Hence, for the small volume organs, we would suggest to evaluate them based on nonoverlapping volume in $\mathrm{cc}$ rather then overlapping index. Besides that, qualitative study on the deformable registration may provide better understanding and evaluation on the software.

Conclusions Deformable registration for certain organs and target like eye, spinal cord, GTV, parotid and brainstem have potential for significantly reducing the clinical turnaround time for re-planning and dose accumulation evaluation in head and neck patient. 